International Journal of Comparative Psychology, 2010, 23, 201-215.

Copyright 2010 by the International Society for Comparative Psychology

\title{
Domesticated Dogs' (Canis familiaris) Response to Dishonest Human Points
}

\author{
Shannon M. A. Kundey \\ Hood College, U.S.A. \\ Andres De Los Reyes \\ University of Maryland at College Park, U.S.A. \\ Jessica Arbuthnot, Rebecca Allen, Ariel Coshun, \\ Sabrina Molina, and Erica Royer \\ Hood College, U.S.A.
}

\begin{abstract}
Pointing is a conventional communicative gesture used by humans to direct others' attention to an environmental feature. Several researchers have argued that pointing becomes so ingrained for humans from a young age that children often have difficulty interpreting the gesture in a novel way. Recent research suggests domestic dogs are also sensitive to human gestures (including points) and proficient in recognizing and acting on humans' visual attention. We explored the role of pointing in dogs' choice behavior and whether dogs, like human children, have difficulty interpreting the gesture novelly. In Experiment 1, we explored whether dogs would differentially follow a static human point when it was administered by a familiar or unfamiliar individual and that individual indicated or failed to indicate the correct location of a food reward. The results indicated dogs chose the container specified by the demonstrators' point in the honest and dishonest condition. Demonstrator familiarity did not alter performance. In Experiment 2, we compared dogs' propensity to follow a static point versus other cues (momentary point, standing location) when the cue never indicated the correct location of a food reward, which was either visible or hidden during choice. The results suggested dogs did not inhibit their approach to a location indicated by a deceptive static point even when the location of a reward was visibly available during choice. However, dogs used a deceptive momentary point or standing location to locate food in both visible and hidden trials. In Experiment 3, we explored if dogs could overcome their tendency to follow a deceptive static point. These results indicated dogs learned to inhibit their approach to a deceptive static point when the reward was visible during choice. However, when information about the reward's location was later hidden, dogs reverted to following the demonstrator's static point.
\end{abstract}

For humans, pointing is a conventional communicative gesture used to direct the attention of others to a feature of their surroundings (Couillard \& Woodward, 1999). At a very early age, human infants are skilled at using points and interpreting the points of others (e.g., Bates, Beningni, Bretherton, Camaionin, \& Volterra, 1979; Franco, \& Butterworth, 1996; Lakatos, Soproni, Dóka, \& Miklósi, 2009; Schaffer, 1984). Some have suggested such pointing has special meaning for humans from very early in their life (Butterworth, 1995; Couillard \& Woodward, 1999) and further that the knowledge of what a point means becomes so ingrained that young humans have difficulty interpreting points in a new way.

We would like to thank Sherry McClurkin, Robin Reutten, and Chelsea Taglang for their assistance in data collection and participant recruitment for this study. Correspondence concerning this article should be addressed to Shannon M.A. Kundey at Hood College, Department of Psychology, 401 Rosemont Avenue, Room ROS 27, Frederick, MD 21701, U.S.A. (kundey@ hood.edu). 
For example, Couillard and Woodward (1999) studied three- and four-year olds' ability to comprehend deceptive point gestures. An experimenter provided misleading cues about the location of a reward by either pointing to or placing a marker on the container without the reward. Overall, children performed poorer when the cue was a point rather than a marker. Children in all age groups were able to use the misleading marker cue, learning to successfully look under the unmarked container for a reward. However, the youngest children included in the study (3 years, 3 months) continued to follow the deceptive point over 10 trials while older children ( 3 years, 8 months and 4 years, 3 months) performed at chance during point trials. Overall, children appear to express sensitivity to the cues suggested by human pointing.

Recent research suggests domestic dogs (Canis familiaris) are also highly sensitive to pointing as well as a variety of other human gestures (e.g., Agnetta, Hare, \& Tomasello, 2000; Hare, Call, \& Tomasello, 1998; McKinley \& Sambrook, 2000; Miklósi, Polgárdi, Topál, \& Csányi, 1998; Soproni, Miklósi, Topál, \& Csányi, 2001, 2002). In these studies, researchers often construct situations in which a human hides food in one of several distinct locations and subsequently provides a cue to the dog to indicate where the food is hidden. Generally, this work indicates that dogs locate hidden food at above-chance levels and via several different human cues. Such cues are diverse and include pointing to the target location, gazing to the target location (head and eyes oriented to the target either dynamically or statically), bowing or nodding to the target location, and placing a marker in front of the target location. Some dogs also show the ability to use an eye glance alone to locate food either spontaneously or following training. Subsequent studies have revealed that dogs can use relatively novel gestures such as pointing with the leg (Lakatos et al., 2009). Interestingly, successful performance is not limited to adult canines, as dogs under six months of age are also skillful in using basic gestural cues (e.g., Agnetta et al., 2000). Dogs also appear more skillful than wolves (Canus lupus) in many tasks involving action based on human gestures (e.g., Frank \& Frank, 1983; Hare, Brown, Williamson, \& Tomasello, 2002; Miklósi, Kubinyi, Topál, Gácsi, Virányi, \& Csányi, 2003), leading some to propose that the ability to understand human-given gestures within a social context is a product of domestication. However, others have found evidence suggesting domestication is not a prerequisite for understanding human gestures (e.g., Udell, Dorey, \& Wynne, 2008; Virányi, Gácsi, Kubinyi, Topál, Belényi, Ujfalussy, et al., 2008).

Recent research with dogs suggests that they, like human children, show difficulty interpreting a human point in a novel (or contradictory) way (Petter, Musolino, Roberts, \& Cole, 2009). In several studies, dogs chose between two containers, only one of which contained a food reward. A cooperative human pointed to the baited container on some trials while a deceptive human pointed to the non-baited container on other trials. As expected, dogs learned to approach the cooperative human more often than the deceptive human. However, they continued to approach the deceiver on many trials despite not receiving a food reward for their approach. The researchers argued that the dogs used the presence of a human 
as a cue to the location of food reward. However, the role of the human indicator's point in this continued approach was not directly evaluated.

Here, we explore the role of pointing in dogs' choice behavior and whether dogs, like human children, have difficulty interpreting the gesture in a new (contradictory) way. In effect, we evaluated whether dogs could inhibit their approach in response to a human demonstrators' pointing cues when the demonstrator indicated an incorrect location of a food reward.

To accomplish this, in Experiment 1 we explored whether dogs would differentially follow a static human point when it was administered by a familiar or unfamiliar individual and that individual indicated or failed to indicate the correct location of a food reward. Importantly, Experiment 1 also allowed us to develop a baseline for dogs' general propensity to follow a point cue in our paradigm, to which we could compare other types of human gesturing. In Experiments 2 and 3, we compared dogs' propensity to follow a point versus other cues when the cue administered never indicated the correct location of a food reward and the reward was either visible or hidden during choice.

\section{Experiment 1}

We first evaluated whether dogs would differentially follow a static human point when it indicated or failed to indicate the location of a food reward. Additionally, we investigated the role of familiarity with the deceiver. We reasoned that dogs might have more experience following the pointing gestures of their owner and would therefore be more likely to continue to follow these gestures than those of an unfamiliar human demonstrator even when not rewarded for this action. We also reasoned that dogs would be more likely to refuse to make choices when incorrect points were administered by an unfamiliar individual.

Evidence suggesting familiarity might play a role in dogs' responses in object choice tasks was obtained recently by Elgier and colleagues (Elgier, Jakovcevic, Mustaca, \& Bentosela, 2009). More specifically, they found that extinction was slower but reversal learning was faster when the owner gave a cue (a static point) compared with an unfamiliar stranger. In their extinction task, dogs encountered trials in which a demonstrator pointed at one of two locations. However, neither location contained food. In their reversal task, dogs encountered trials in which a demonstrator pointed at one of two locations. However, the nonindicated location contained food. The reversal task involved two phases. First, dogs were first physically led to the non-indicated location by a handler while the demonstrator pointed at the unbaited container during an "instigation" phase to aid dogs in learning that the reinforcer was not contained in the container the demonstrator indicated. In subsequent reversal trials, dogs were allowed to make choices between a non-indicated container containing food and an indicated container not containing food. Choices to the non-indicated container were rewarded while those to the indicated container were corrected.

In Experiment 1, we sought to evaluate whether dogs would differentially follow a static human point when it was administered by a familiar or unfamiliar 
individual and indicated or failed to indicate the location of a food reward absent extinction or instigation training like that employed by Elgier and colleagues (Elgier et al., 2009). We felt this was important as it allowed us to develop a baseline for dogs' general propensity to follow a pointing cue in our paradigm to which we could then compare other types of human gesturing. To this end, a demonstrator, who was either honest (always unfamiliar to the dog) or dishonest (familiar or unfamiliar to the dog), gained the dog's attention and displayed a treat. Next, the demonstrator secretly hid the treat in one of two containers. In the honest condition, the demonstrator moved from a neutral position to statically point to the correct location of the treat. In the dishonest condition, the demonstrator moved from a neutral position to statically point to the incorrect location. The dog was then allowed to freely approach the containers, and the demonstrator gave the dog the contents of the first container to which the dog approached. If the dog picked the empty container, the experimenter retrieved the treat from the correct container, showed it to the dog, and pretended to eat it. The honest demonstrator was always an unfamiliar human experimenter, but the dishonest demonstrator was either an unfamiliar human experimenter or the dog's owner. When the owner served as the demonstrator, an experimenter held the dog at the start position.

Importantly, recent work by Fiset, Beaulieu, and Landry (2003) indicates that dogs' can accurately locate hidden objects at levels above-chance after up to a 240 s delay. Thus, it seems unlikely that any inability to find the food reward would be indicative of a memory deficit for the food's actual location after a short delay. Additionally, many researchers have investigated dogs' abilities to infer the location of a reward (e.g., Collier-Baker, Davis, \& Suddendorf, 2004; Watson, Gergely, Csanyi, Topal, Gacsi, \& Sarkozi, 2001). Although many such studies have concluded it is likely that dogs solve classical tasks of inference like invisible displacement through associative mechanisms, others have found evidence of more sophisticated inference abilities (e.g., Aust, Range, Steurer, \& Huber's 2008 work on reasoning by exclusion).

\section{Method}

\section{Subjects}

Owners and their dogs were recruited through personal contact with the laboratory staff or via fliers in the community. Participation was voluntary and limited to dogs over one year of age. Dogs had not received any advanced obedience training. Their owners also reported them to be free from major health problems (e.g., arthritis). Laboratory staff pointed out to the owners that there were no right or wrong behaviors and that they were not to help their dog.

As described below, before exposure to experimental trials all dogs completed pretraining to ensure they would make choices freely from two containers. Sixteen dogs $(M=4.7$ years, $S D=$ 2.8, 7 females, 9 males) successfully completed this pretraining and were included in the study. Two dogs were excluded due to a side bias during pretraining (i.e., making choices from only one of two possible containers); 1 additional dog was excluded due to failure to acclimate to the laboratory room prior to pretraining (e.g., excessive barking or misbehavior that could not be reduced or eliminated by owner). 


\section{Apparatus}

Two plastic, opaque green cylindrical containers were employed $(38 \mathrm{~cm}$ tall; $22 \mathrm{~cm}$ diameter). The bottom of each container contained approximately $2.5 \mathrm{~cm}$ of cotton batting secured by duct tape. The containers were false-baited throughout pretraining and all experimental trials to control for possible use of olfactory cues. False-baiting was accomplished by placing 20 dog treats below the cotton batting in each container. The insides of each container were also rubbed with treats frequently. Commercial dog treats $(3 \mathrm{~cm} \mathrm{X} 1.25 \mathrm{~cm})$ were used as rewards. A large opaque tablecloth was also employed $(150 \mathrm{~cm}$ X $150 \mathrm{~cm})$.

\section{Procedure}

All procedures were approved by the college's Animal Care and Use Committee. Testing occurred in a room within the laboratory. The dog's owner was present throughout all testing sessions.

First, dogs were allowed to become familiar with test apparatus and their general surroundings by exploring unhindered while their owner completed various forms regarding the dog's general health, disposition, and training (approximately 10-15 min). Following this acclimation period, all dogs underwent pretraining.

The goal of pretraining was to accustom the dog to the containers. We permitted the owner to make short general commands to stay, sit, and release. During pretraining, the containers were positioned $1.5 \mathrm{~m}$ apart, and the dog was positioned to sit on a floor marking approximately $3 \mathrm{~m}$ equidistant from the containers.

The experimenter then stood in the middle of the containers in front of the dog and showed a treat to the dog. As the dog watched, she placed the treat inside one of the two opaque containers (counterbalanced across subjects). She then returned to a neutral position equidistant from both containers and looked straight ahead. The dog was then released and allowed to retrieve the treat. During the choice, the experimenter continued to look straight ahead.

If the dog chose the correct container, it was given the treat. If the dog chose the incorrect container, the experimenter left the center position and moved to the baited container to retrieve the treat. She then showed the dog the treat and pretended to eat it. The experimenter then moved to the next pretraining trial. Dogs received only two pretraining trials for each container. Only dogs that successfully completed pretraining by correctly locating food reward in all four pretraining trials continued to the experimental phase.

Two types of test trials were conducted: "honest" and "dishonest". For the test trials, the containers were positioned $1.5 \mathrm{~m}$ apart from each other and $3 \mathrm{~m}$ in front of the dog. The demonstrator stood behind and between the containers in front of the dog. Both the dog and demonstrator were equidistant from both containers. In this experiment, one unfamiliar demonstrator gave accurate information (honest trials), whereas another demonstrator gave inaccurate information (dishonest trials). The honest demonstrator was always an unfamiliar person to the dog. However, for one group (8 dogs) the dishonest demonstrator was a second unfamiliar person to the dog while for another group ( 8 dogs) the dishonest demonstrator was their owner.

In honest trials, the demonstrator gained the dog's attention and displayed a treat. The dog's view was then hidden by a tablecloth or by covering its eyes while the demonstrator placed the treat in one of the two containers (counterbalanced across trials and subjects). Next, she returned to stand behind the containers, equidistant from both. When she had returned to this neutral position, the tablecloth was lowered or the dog's eyes were uncovered. Then, with the dog watching, she moved to stand behind the container bearing the treat. She bent at the waist and used her index finger to point at the container holding the treat such that the tip of her finger was approximately $12 \mathrm{~cm}-24 \mathrm{~cm}$ above the container's rim. The dog was then allowed to make a choice. A choice was defined as the first container the dog approached within approximately $5 \mathrm{~cm}$ of its snout. If the dog chose correctly (i.e., picked the container the treat was in), it was allowed to consume the treat. If the dog chose incorrectly (i.e., picked the container not holding the treat), the demonstrator removed the treat from the correct container. She showed it to the dog and then pretended to consume it. If dogs failed to make a choice within $60 \mathrm{~s}$ or lay down, a "no choice" response was recorded. The dishonest trials 
exactly resembled the honest trials except that the demonstrator pointed to the container the treat was not in.

Trials were run in 4 blocks of 6 trials each in the following order: "honest block 1", "dishonest block 1", "dishonest block 2" and "honest block 2". The trials were blocked in this manner so we could first ensure dogs used the static pointing gesture to find food before providing incorrect information in the dishonest blocks. A second block of honest trials was run following the two sets of dishonest trials to ensure dogs were still motivated to find food (e.g., that they had not become satiated) and to relieve any discomfort induced by the dishonest trials.

\section{Data analysis}

Digital videos (30 frames/s) were analyzed using Adobe Premiere Elements7.0 software by naïve coders. Responses were counted as correct only when the dog located the treat on the first choice. The maximum time dogs were allocated to make a choice was $60 \mathrm{~s}$. One-quarter of all trials were scored by an additional coder (interobserver reliability: $\kappa=0.96$ ). We then employed appropriate repeated measures Analyses of Variance (ANOVA) and post hoc tests to examine dogs' choices. Effects were considered significant only if $p<0.05$.

\section{Results and Discussion}

Dogs' choices are depicted in Figure 1. To analyze the choices dogs made, we first conducted a Group (familiar vs. unfamiliar dishonest demonstrator) X Trial Type (honest vs. dishonest) X Block mixed model ANOVA. This indicated a main effect for trial type $(F(1,14)=2693.10, p<0.01)$ and block $(F(1,14)=5.73$, $p=0.03)$. No interactions were significant at the $p<0.05$ level. Next, we conducted paired-sample $t$ tests to further explore these effects. We compared the number of correct choices overall in the honest and dishonest conditions. This revealed that dogs chose correctly significantly more often in the honest condition than the dishonest condition $(t(15)=51.43, p<0.01)$. However, no significant differences were seen between dogs' pattern of choices for the first block of honest trials and the second block of honest trials $(t(15)=1.46, p=0.16)$, nor between the first block of dishonest trials and the second block of dishonest trials $(t(15)=1.73$, $p=0.10$ ). This suggests that dogs' choices were consistent across trials, indicating a lack of a learning effect for both the honest and dishonest conditions.

Finally, we compared the number of trials in which dogs did not make choices using a Group (familiar vs. unfamiliar dishonest demonstrator) X Trial Type (honest vs. dishonest) mixed model ANOVA. This revealed a significant main effect of Group $(F(1,14)=5.94, p=0.03)$ and Trial Type $(F(1,14)=8.57$, $p=0.01)$. Additionally, a significant Group $\mathrm{X}$ Trial Type interaction was found $(F(1,14)=5.13, p=0.04)$. Subsequent independent-samples $t$ tests indicated that dogs made significantly more no choice responses when an unfamiliar person gave incorrect information than when their owner gave incorrect information $(t(8)=$ $2.39, p=0.04)$. Thus, in the absence of definitive information on the location of the treat, dogs nevertheless were more likely to enact a response in reference to their owner relative to an unfamiliar person.

Overall, dogs correctly chose the container holding the treat in the honest condition but not the dishonest condition. In both conditions, dogs chose the container indicated by the demonstrators' point significantly more often than 
chance. However, dogs only made no choice responses in the dishonest condition when the demonstrator was an unfamiliar individual. Dogs' poor performance in the dishonest condition suggests they were not relying on differential olfactory or auditory information, if it was available, to locate treats. These results suggest that dogs readily follow a human's point cue, even when the cue does not provide useful information on locating a food reward and the person administering the cue is an unfamiliar individual. However, the procedure that we adopted in this experiment might have biased dogs to follow a demonstrator's point. More specifically, all dogs first encountered an honest demonstrator before encountering a dishonest demonstrator. Additionally, dogs were only given a small number of trials within Experiment 1. We sought to resolve these issues, as well as compare the static pointing cue used in Experiment 1 to other possible cues in Experiments 2 and 3 .

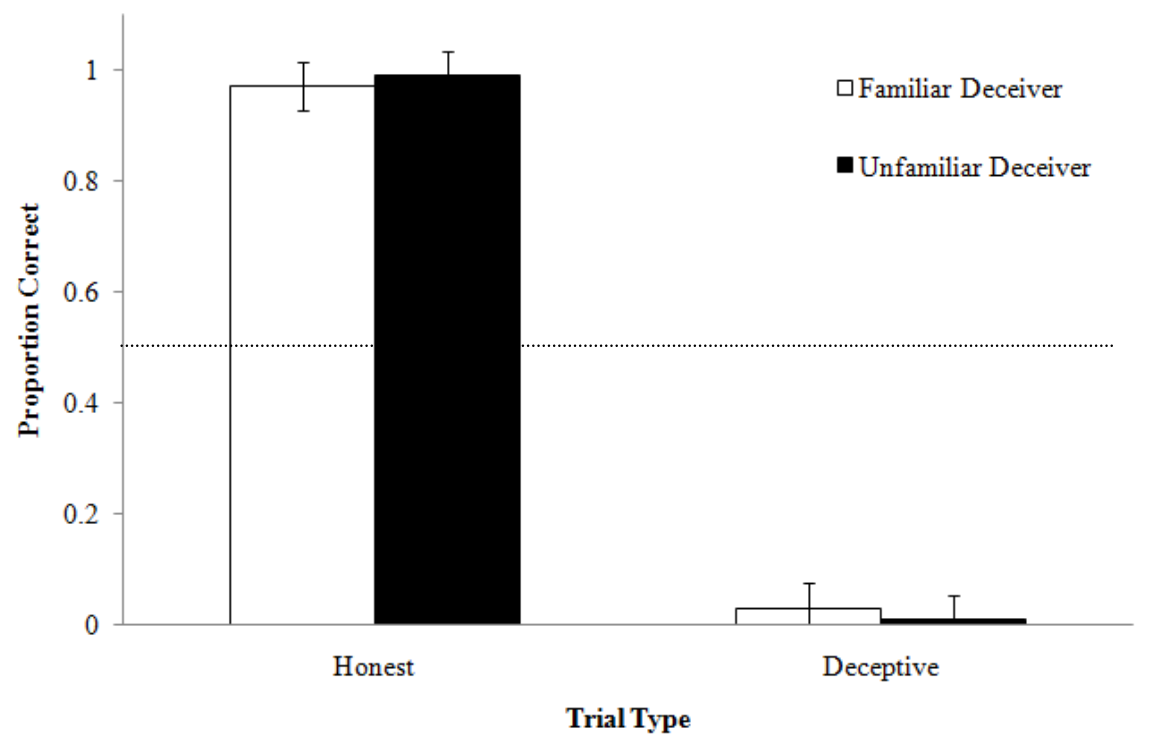

Figure 1. Proportion of correct choices for honest and dishonest trials in Experiment 1 collapsed across blocks. Results are depicted by whether the dog was deceived by an unfamiliar demonstrator or their owner in the dishonest trials. Honest trials were always conducted by an unfamiliar demonstrator. Dashed line represents chance responding.

\section{Experiment 2}

The purpose of Experiment 2 was twofold. First, we wanted to explore whether dogs would continue to approach a non-baited container indicated by a deceptive demonstrator through pointing even when they could visually see the contents of the baited and unbaited container throughout the choice trial. Second, we compared a demonstrator's static pointing to the unbaited container (static point), momentarily pointing to the unbaited container (momentary point), - 207 - 
standing behind the unbaited container (stand), as well as a condition in which the demonstrator gave no cues regarding the baited location (no cue). Given that in Experiment 1 dogs overall were able to correctly locate treats in honest trials, Experiment 2 only employed dishonest trials.

\begin{abstract}
Method
Subjects

The subjects were similar to those included in Experiment 1 and were recruited in a similar manner. In total, 32 dogs ( $M=3.8$ years, $S D=3.1,17$ females, $)$ successfully completed pretraining and were included in the study. One dog was excluded due to nervousness prior to pretraining, and a second was excluded due to food aggression during pretraining (i.e., research personnel determined there were safety concerns in continuing trials with this dog).
\end{abstract}

\title{
Apparatus
}

Two rectangular clear glass containers were employed $(10 \mathrm{~cm} \mathrm{X} 10 \mathrm{~cm} \mathrm{X} 22 \mathrm{~cm})$. A thin swath of white foam was placed in the bottom of each container $(10 \mathrm{~cm} \mathrm{X} 10 \mathrm{~cm} \mathrm{X} 0.5 \mathrm{~cm})$. This heightened the contrast between the brown dog treats used and the containers' bottoms. The containers were false-baited throughout pretraining and all experimental trials to control for the possible use of olfactory cues. False-baiting was accomplished by securing dog treats to the underside (non-visible portion) of the white foam located at the bottom of each container. Additionally, the insides of each container were rubbed with treats frequently. Two opaque, florescent pink cylindrical occluders were also utilized $(22 \mathrm{~cm}$ tall $X 7.5 \mathrm{~cm}$ circumference). We also employed a large rectangular, black board $(50 \mathrm{~cm} \mathrm{X} 75 \mathrm{~cm})$. Similar commercial dog treats to those employed in Experiment 1 were also used.

\section{Procedure}

Pretraining and experimental procedures were similar to Experiment 1 with several exceptions. First, after pretraining, only dishonest experimental trials were conducted and the demonstrator was always an unfamiliar experimenter. For each group, 2 blocks of 6 trials were conducted: Visible and Hidden. Visible trials were always conducted first, followed by hidden trials. In all trials, the dog was positioned $1.5 \mathrm{~m}$ from the containers.

In visible trials, dogs watched as the demonstrator displayed a treat and visited both containers. However, she only deposited a treat into one container. The location of the treat was visible to the dog through the clear walls of the container. The demonstrator was careful to act equally on each container. Next, she returned to stand equidistant from both containers. Then, she displayed one of four actions depending on the dog's group membership ( 8 dogs per group): static point, momentary point, stand, or no cue. The static point exactly resembled the static point described in Experiment 1. The momentary point resembled the static point with the exception that the demonstrator stood between the two containers and pointed with the arm closest to the unbaited container for approximately $1 \mathrm{~s}$ before looking straight ahead. In the stand condition, the demonstrator simply stood behind the incorrect container while looking straight ahead. Finally, in the no cue condition, the demonstrator continued to stand between the two containers while looking straight ahead. Following the demonstrator's cue, the dog was allowed to choose a container; treats were dispensed as in Experiment 1.

Hidden trials resembled visible trials with the exception that pink opaque occluders were inserted within the clear containers, making it impossible for the dog to see where the treat was located through the walls of the container. In these trials, dogs saw the demonstrator display a treat. The demonstrator then moved the treat behind the large board. She then closed her fist around the treat to further ensure the treat was obscured from view. She moved to visit each container, lowering her closed fist into each. During one visit, she deposited the treat. However, before removing her hand from the container, she again closed her fist. Thus, it was impossible to tell from the 
demonstrator's actions where the treat was deposited as the treat was always hidden from view. Next, she displayed one of four actions depending on the dog's group membership as described above and the dog was allowed to make a choice. Treats were dispensed as in Experiment 1.

Data analysis

All experimental trials were recorded and analyzed in a similar manner to Experiment 1. Interrater reliability was $\kappa=0.93$.

\section{Results and Discussion}

Figure 2 depicts dogs' choices in each condition for the two trial types. To analyze dogs' choices, we first conducted a Group (static point, momentary point, stand, or no cue) $\mathrm{X}$ Trial Type (visible vs. hidden) mixed model ANOVA. This indicated a main effect for group $(F(3,28)=20.39, p<0.01)$ and trial type $(F(1,28)$ $=14.50, p<0.01)$. No interactions were significant at the $p<0.05$ level. This suggested that overall dogs chose correctly more often in the visible than hidden trials. Subsequent independent-samples $t$ tests indicated that the static point group differed significantly from all other groups for both visible (stand: $t(14)=2.63, p=$ 0.04; momentary point: $t(14)=3.19, p<0.01$; no cue: $t(14)=2.29, p=0.04$ ) and hidden trials (stand: $t(14)=9.32, p<0.01$; momentary point: $t(14)=8.22, p<$ 0.01 ; no cue: $t(14)=7.10, p<0.01$ ). However, the remaining groups did not differ significantly from each other at the $p<0.05$ level. Next, we explored the performance of dogs in each condition using one-sample $t$ tests for visible and hidden trials. In visible trials, the momentary point $(t(7)=5.23, p<0.01)$, stand $(t(7)=7.18, p<0.01)$, and no cue $(t(7)=3.97, p<0.01)$ groups successfully located the food reward at above-chance levels while the static point $(t(7)=0.74, p$ $=0.48)$ group did not. In hidden trials, only subjects in the momentary point $(t(7)=$ $2.50, p=0.04)$ and stand $(t(7)=2.48, p=0.04)$ conditions successfully located the food reward at above-chance levels. In hidden trials, the no cue group did not significantly differ from chance $(t(7)=1.83, p=0.11)$, while the static point group chose the container indicated by the demonstrator at above-chance levels $(t(7)=$ $16.99, p<0.01)$.

Overall, dogs correctly chose the baited container when information about which container the treat was in was visibly available during their choice after watching the demonstrator momentarily point to the unbaited container, stand behind the unbaited container, and give no cues. However, dogs watching the demonstrator statically point at the non-baited container did not correctly choose the container containing the treat in visible trials even though information regarding the treat's location was visibly available to them at the time of choice. Dogs in the momentary point and stand conditions were able to continue correctly choosing the treat's location in hidden trials when they were not given direct information about where the treat was placed nor able to see the treat's location during their choice during hidden trials. However, dogs were unable to choose the correct location in the no cue or static point conditions in hidden trials. During these trials, dogs in the static point condition chose the container indicated by the demonstrator's point while dogs in the no cue condition chose randomly. Dogs' 
performance in the no cue condition in hidden trials suggests that they were not relying on differential olfactory information, if it was available, to locate treats. Additionally, dogs' performance in the visible and hidden static point condition, as well as dogs' performance in the no cue condition suggests they were not relying on differential auditory information, if it was available, to locate treats.

These results suggest dogs have difficulty inhibiting their approach to a location indicated by a static point even when the location of a reward is visibly available to them during choice. Given these results, we wondered whether dogs could learn to overcome their tendency to follow a static point when the location of the treat was available to them during choice if given more experience in the task.

\section{Experiment 3}

In Experiments 1 and 2, dogs showed difficulty inhibiting behavior to a unbaited location indicated by a static pointing gesture-even when the location of the food reward was visibly available to them during choice. In Experiment 3, we explored whether dogs could overcome their tendency to follow a static point if given additional trials.

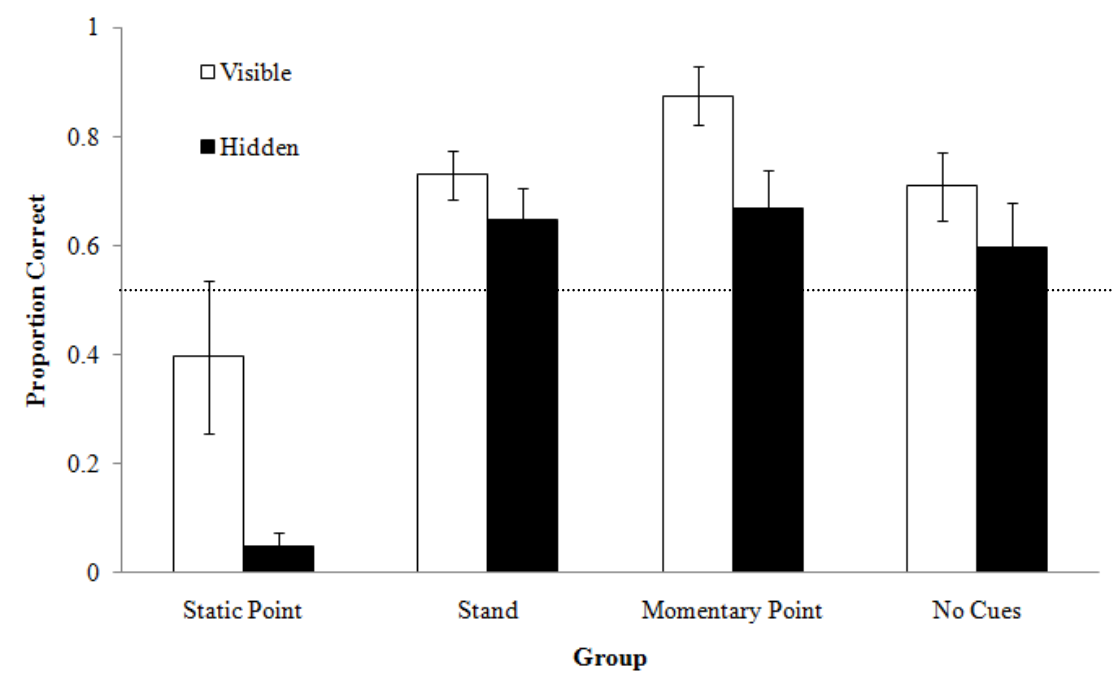

Figure 2. Proportion of correct choices for visible and hidden trials in Experiment 2. Results are depicted by group: static point, momentary point, stand, or no cue. Dashed line represents chance responding.

Method

Subjects

The subjects were similar to those included in Experiments 1 and 2 and were recruited in a similar manner. In total, 8 dogs $(M=3.9$ years, $S D=2.5,3$ females successfully completed pretraining and were included in the study. All subjects successfully completed pretraining and experimental trials. 
The containers, pink occluders, and large rectangular board from Experiment 2 were employed, along with similar commercial dog treats.

\section{Procedure}

Pretraining and experimental procedures were similar to those employed in Experiment 2 with the exception that 24 trials were run in the visible condition and 6 trials in the hidden condition. All visible trials were run prior to hidden trials. Additionally, only the static point group was included. The demonstrator was always an unfamiliar experimenter.

\section{Data analysis}

All experimental trials were recorded and analyzed in a similar manner to Experiment 1. Interrater reliability was $\kappa=0.94$.

\section{Results \& Discussion}

Dogs' choices in Experiment 3 are depicted in Figure 3. We first divided the 24 visible trials into 4 blocks of 6 trials each. We then conducted a repeated measures ANOVA with block as a within-subjects variable. This revealed a significant main effect of block $(F(1,7)=30.74, p<0.01)$. This indicated that dogs improved their accuracy with repeated trials. Next, we conducted one-sample $t$ tests for each block to determine when dogs first began to perform at above-chance levels. These tests indicated that dogs located the treat significantly above chance beginning in block $3(t(7)=3.27, p=0.01)$ and continued this performance in block $4(t(7)=6.36, p<0.01)$. These results indicate that dogs can, with enough practice, learn to inhibit following a static pointing gesture when the reward is visible during choice.

Next, we evaluated dogs' performance during hidden trials using a onesample $t$ test. This indicated that dogs did not perform significantly better than chance when the treat was not visible during choice. Instead, they reverted to choosing the location indicated by the demonstrator's static point $(t(7)=3.87, p=$ $0.01)$. This suggests that when information about the treat's location is no longer visible during choice, dogs reverted to following the demonstrator's pointing gesture. 


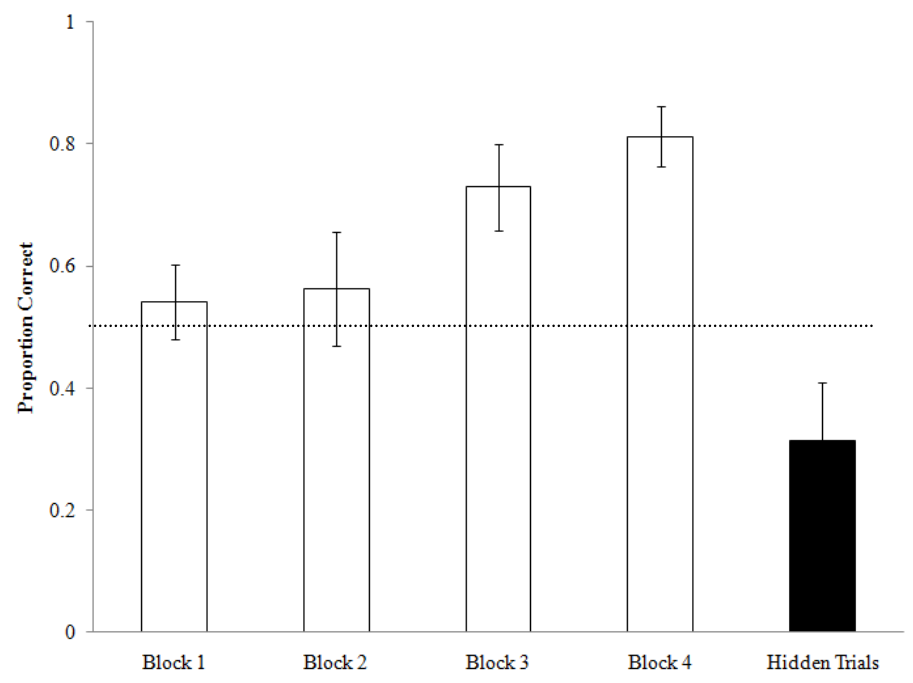

Figure 3. Proportion of correct choices for visible and hidden trials in Experiment 3. Results are depicted by block. Dashed line represents chance responding.

\section{General Discussion}

In three experiments, we explored the role of human gesturing, namely static points, in dogs' choice behavior. In particular, we explored whether dogs, like human children (Couillard \& Woodward, 1999), would have difficulty interpreting a static pointing gesture emitted by a human in a new (contradictory) way and if so, under which conditions.

Past research with dogs has indicated that they are highly sensitive to a variety of human gestures, including static points (e.g., Agnetta et al., 2000; McKinley \& Sambrook, 2000; Soproni et al., 2001, 2002). However, most studies have focused on the role of honest gestures. For example, in a study employing honest and deceptive static points, Petter and colleagues (2009) found that dogs learned to approach the cooperative human more often than the deceptive human. However, dogs continued to approach the deceiver on many trials despite not receiving a food reward for their approach, suggesting that they, like young human children (Couillard \& Woodward, 1999), have difficulty interpreting a static point in a novel way. However, the role of the human indicator's static point in this continued approach was not directly evaluated by Petter and colleagues (2009). Our experiments differ from those of Petter and colleagues in that we sought to directly evaluate the role of the static point specifically and dissociate the static point gesture from the mere presence of a human as a cue for food.

First, we were specifically interested in whether dogs' familiarity with the deceiver would affect performance. We reasoned that dogs might have more experience following the pointing gestures of their owner and would therefore be more likely to continue to follow deceptive gestures from their owner than from an unfamiliar demonstrator. We explored this issue in Experiment 1 by varying 
demonstrator familiarity. For some dogs, the deceiver was very familiar to them (their owner), whereas for other dogs the deceiver was an unfamiliar person (an experimenter). The results of the experiment indicated that dogs chose the container indicated by the demonstrators' deceptive points regardless of whether the demonstrator was familiar or unfamiliar to them. However, dogs were more likely to refuse to make a choice when an unfamiliar individual served as the deceiver.

While we did not directly explore why dogs were more likely to refuse to make choices for an unfamiliar demonstrator, it is possible that dogs' perception of the context of their task differed between groups. For instance, it is possible that dogs interpreted their owner's actions as part of a game (potentially leading to higher engagement), but did not make this same interpretation for the unfamiliar demonstrator. Overall, these results suggest that dogs readily follow a human's static point cue, even when the cue does not provide useful information to locate a food reward, regardless of familiarity with the deceiver. This expands on the work of Petter and colleagues (2009) in that their demonstrators were always unfamiliar to the dog.

Additionally, we expanded on Petter and colleagues' (2009) work by exploring the role of multiple cue types (static point, stand behind unbaited container, momentary point) in Experiment 2 when the treat was both visually available and hidden. We compared performance for both visible and hidden trials to a control condition in which the demonstrator gave no cues regarding the location of the food reward (no cue). While dogs were able to choose the baited container when information about which container the treat was in was visually available at choice in the momentary point, stand, and no cues condition, dogs were unable to perform at an above-chance level in the static point condition. When dogs could not detect the location of the treat visually through the containers' walls, dogs maintained their successful performance in the momentary point and stand conditions. However, they performed at chance in the no cues condition and reverted to following the demonstrator's gesture in the static point condition. The results suggested dogs had difficulty inhibiting their approach to a location indicated by a static point even when they could see the location of the treat by looking through the containers' walls. This experiment is informative in that it provides additional evidence that dogs' use of static points differs from the other gesture types explored here. In particular, dogs' difficulty inhibiting approach directed by a static point in comparison to the stand condition in which the demonstrator's body position indicated the unbaited container suggests the static point itself offers information to the dog beyond what is offered by a human's body position alone.

Given dogs' difficulty inhibiting following of dishonest human points, we explored in Experiment 3 whether additional training would improve dogs' performance. Our results indicated dogs did learn, with additional practice, to inhibit following of a static point gesture when the location of the food reward was visible through the containers' walls. However, when the location of the food reward was obscured by an opaque barrier in each container, dogs reverted to 
following a humans' static point gesture. Future research might explore whether substantially more training when the treat can be visually located through the containers' walls might improve later performance when the location of the treat is made visually unavailable at the time of choice.

In conjunction, this work suggests that dogs, like human children (Couillard \& Woodward, 1999), do have difficulty interpreting static points in a new way in comparison to other types of human cuing (e.g., momentarily pointing and body position). In sum, these findings extend the literature on human and nonhuman animals in the role of gesturing in choice behavior and suggest that even in the presence of conflicting information dogs are heavily influenced by where human gestures indicate the presence of food rewards.

\section{References}

Agnetta, B., Hare, B., \& Tomasello, M. (2000). Cues to food locations that domestic dogs (Canis familiaris) of different ages do and do not use. Animal Cognition, 3, 107112.

Aust, U., Range, F., Steurer, M., \& Huber, L. (2008). Inferential reasoning by exclusion in pigeons, dogs, and humans. Animal Cognition, 11, 587-597

Bates, E., Benigni, L., Bretherton, I., Camaioni, L., \& Volterra, V. (1979). The emergence of symbols: Cognition and communication in infancy. New York: Academic Press.

Butterworth, G. (1995). Origins of mind in perception and action. In C. Moore \& P. J. Dunham (Eds.), Joint attention: Its origins and role in development (pp. 29-40). Hillsdale, NJ: Lawrence Erlbaum.

Collier-Baker, E., Davis, J., \& Suddendorf, T. (2004). Do dogs (Canis familiaris) understand invisible displacement? Journal of Comparative Psychology, 118, 421433.

Couillard, N., \& Woodward, A. (1999). Children's comprehension of deceptive points. British Journal of Developmental Psychology, 17, 515-521.

Elgier, A., Jakovcevic, A., Mustaca, A., \& Bentosela, M. (2009). Learning and ownerstranger effects on interspecific communication in domestic dogs (Canis familiaris). Behavioural Processes, 81, 44-49.

Fiset, S., Beaulieu, C., \& Landry, F. (2003). Duration of dogs' (Canis familiaris) working memory in search for disappearing objects. Animal Cognition, 6, 1-10.

Franco, F., \& Butterworth, G. (1996). Pointing and social awareness: Declaring and requesting in the second year. Journal of Child Language, 23, 307-336.

Frank, H., \& Frank, M. (1983). Inhibition training in wolves and dogs. Behavioural Processes, 8, 363-377.

Hare, B., Brown, M., Williamson, C., \& Tomasello, M. (2002). The domestication of social cognition in dogs. Science, 298, 1634-1636.

Hare, B., Call, J., \& Tomasello, M. (1998). Communication of food location between human and dog (Canis familiaris). Evolution of Communication, 2, 137-159.

Lakatos, G., Soproni, K., Dóka, A., \& Miklósi, Á. (2009). A comparative approach to dogs' (Canis familiaris) and human infants' understanding of various forms of pointing gestures. Animal Cognition, 12, 621-631.

McKinley, J., \& Sambrook, T.D. (2000). Use of human-given cues by domestic dogs (Canis familiaris) and horses (Equus caballus). Animal Cognition, 3, 13-22. 
Miklósi, Á., Kubinyi, E., Topál, J., Gácsi, M., Virányi, Z., \& Csányi, V. (2003). A simple reason for a big difference: Wolves do not look back at humans, but dogs do. Current Biology, 13, 763-766.

Miklósi, Á., Polgárdi, R., Topál, J., \& Csányi, V. (1998) Use of experimenter-given cues in dogs. Animal Cognition, 1, 113-121.

Petter, M., Musolino, E., Roberts, W. A., \& Cole, M. (2009). Can dogs (Canis familiaris) detect human deception? Behavioural Processes, 82, 109-118.

Schaffer, H. R. (1984). The child's entry into a social world. London: Academic Press.

Soproni, K., Miklósi, Á., Topál, J., \& Csányi, V. (2001). Comprehension of human communicative signs in pet dogs. Journal of Comparative Psychology, 115, 122126.

Soproni, K., Miklósi, Á., Topál, J., \& Csányi, V. (2002). Dogs' (Canis familiaris) responsiveness to human pointing gestures. Journal of Comparative Psychology, 116, 27-34.

Udell, M., Dorey, N., \& Wynne, C. (2008). Wolves outperform dogs in following human social cues. Animal Behaviour, 76, 1767-1773.

Virányi, Z., Gácsi, M., Kubinyi, E., Topál, J., Belényi, B., Ujfalussy, D., et al. (2008). Comprehension of human pointing gestures in young human-reared wolves (Canis lupus) and dogs (Canis familiaris). Animal Cognition, 11, 373-387.

Watson, J., Gergely, G., Csanyi, V., Topal, J., Gacsi, M., \& Sarkozi, Z. (2001). Distinguishing logic from association in the solution of an invisible displacement task by children (Homo sapiens) and dogs (Canis familiaris): Using negation of disjunction. Journal of Comparative Psychology, 115, 219-226. 